\title{
Novel Bienzymatic Catalysis System for Oxidative Polymerization of Phenols
}

\author{
Hiroshi Uyama, Hideraru Kurioka, and Shiro Kobayashi ${ }^{\dagger}$ \\ Department of Materials Chemistry, Graduate School of Engineering, \\ Tohoku University, Aoba, Sendai 980-77, Japan
}

(Received August 28, 1996)

KEY WORDS Bienzymatic System/Peroxidase / Glucose Oxidase / Oxidative Polymerization / Phenol /

Enzymatic polymerizations have received much attention as a new methodology for polymer syntheses. ${ }^{1,2}$ Characteristic properties of enzymes are expected to afford polymers with high selectivity and/or novel structure. Recently, enzymatic syntheses of polyaromatics have been developed. ${ }^{3-12}$ Peroxidases or oxidases induced an oxidative polymerization of phenol derivatives, yielding a new class of polyphenols with high thermal stability. ${ }^{7-9}$ Their structures were often very complicated; the polymers from phenol and alkylphenols were mainly composed of a mixture of phenylene and oxyphenylene units (Scheme 1). ${ }^{5-9}$ The molecular weight and solubility of the resulting polymers could be controlled by changing the reaction conditions. This enzymatic process is expected to be an alternative for preparation of phenol polymers without using formaldehyde, which is a monomer for production of conventional phenolic resins (phenol-formaldehyde resins). ${ }^{13}$

Oxidoreductases are often used for quantitative determinations of numerous compounds in various fields due to their high substrate specificity. A combination of cholesterol oxidase and horseradish peroxidase (HRP) was successfully used for accurate determination of cholesterol in organic solvents. ${ }^{14}$ In this bienzymatic system, cholesterol oxidase oxidized cholesterol to produce hydrogen peroxide, then a chromogenic substrate was oxidized by HRP with hydrogen peroxide to give a colored detectable compound.

A peroxidase-catalyzed oxidative polymerization utilizes hydrogen peroxide as oxidizing agent. In case of phenol monomer, the dropwise addition of hydrogen peroxide to the monomer solution during the polymerization is necessary to obtain the polymer in high yields. The present paper describes a new bienzymatic catalysis system (glucose oxidase plus HRP) for the polymerization of phenol derivatives. This system employs hydrogen peroxide which is in situ formed by the oxidation of glucose using glucose oxidase.

\section{EXPERIMENTAL}

Phenol was purified by distillation. Other reagents and enzymes were used as received. Gel permeation chromatographic (GPC) analysis was carried out using a Tosoh SC8010 apparatus with a refractive index (RI) detector under the following conditions: TSKgel $\mathrm{G}_{4000 \mathrm{H}_{\mathrm{HR}}}$ or $\mathrm{G} 2500 \mathrm{H}_{\mathrm{HR}}$ column and DMF containing $0.02 \mathrm{M} \mathrm{LiCl}$ eluent at a flow rate of $0.5 \mathrm{~mL} \mathrm{~min} .^{-1}$ The calibration curves for GPC analysis were obtained using polystyrene standards. IR spectra were recorded on Shimadzu IR-460 spectrometer.

\section{Oxidative Polymerization of Phenol Derivatives by Bienzymatic System}

A typical run was as follows (entry 6 in Table I). To HRP $(10 \mathrm{mg})$, glucose oxidase $(10 \mathrm{mg})$, and phenol $(0.47 \mathrm{~g}, 5.0 \mathrm{mmol})$ in $5 \mathrm{~mL}$ of succinate buffer $(\mathrm{pH} 5.5)$, $15 \mathrm{~mL}$ of 1,4-dioxane was added. The polymerization was initiated by the addition of glucose $(0.91 \mathrm{~g}, 5.0$ $\mathrm{mmol}$ ) in $5 \mathrm{~mL}$ of the buffer. The mixture was stirred at room temperature under air. After $48 \mathrm{~h}$, the solvent was evaporated under reduced pressure. The residue was washed successively with water and methanol, followed by drying in vacuo to give $0.32 \mathrm{~g}$ of the polymer (yield $68 \%$ ).

\section{RESULTS AND DISCUSSION}

Glucose oxidase catalyzes an oxidation of glucose in the presence of oxygen, yielding gluconolactone and hydrogen peroxide (Scheme 2). ${ }^{15}$ Previous studies on the

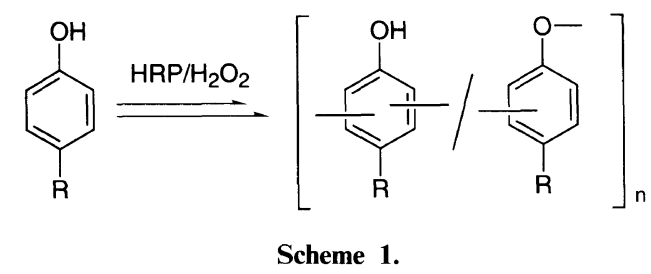

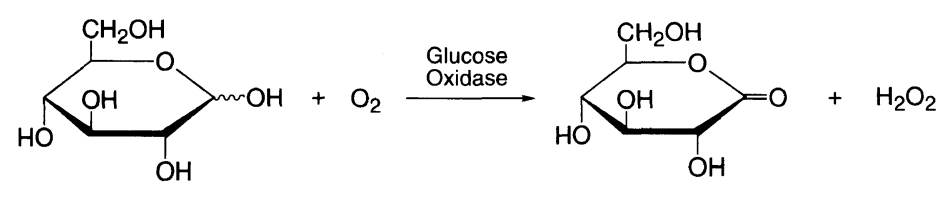

Scheme 2.

\footnotetext{
† To whom correspondence should be addressed.
} 
enzymatic polymerization of phenols revealed that a mixture of a water-miscible solvent and a buffer, e.g., an aqueous dioxane, was suitable as solvent to achieve the high yield and/or with high molecular weight of the polymer. In this study, the addition of glucose and glucose oxidase to HRP-catalyzed polymerization system of phenols has been attempted (Scheme 3). In this study, hydrogen peroxide is not added, instead it is generated in situ.

The polymerization of phenol was carried out in an aqueous 1,4-dioxane at room temperature for $48 \mathrm{~h}$. At first, the effects of the polymerization parameters, i.e., the buffer $\mathrm{pH}$, the mixed ratio of 1,4-dioxane and a buffer, and reaction atmosphere, were examined (Table I). In all cases examined, the present bienzymatic catalysis system afforded the polymer, which was partly soluble in $N, N$-dimethylformamide (DMF) and dimethyl sulf-

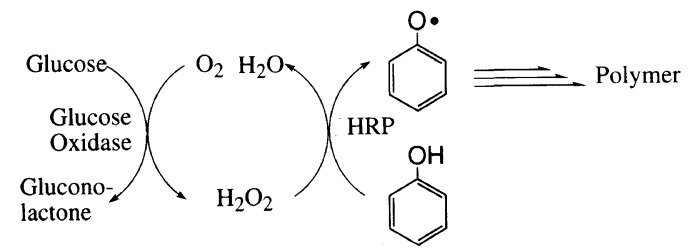

Scheme 3. oxide. The ratio of DMF-soluble part (DSP) was dependent on the polymerization conditions, mainly the mixed ratio of 1,4-dioxane and a buffer. The polymer structure was confirmed by IR spectroscopy; the spectrum pattern of the present polymer was similar to that of the polymer obtained by adding hydrogen peroxide as oxidizing agent, indicating that the present polymer was also composed of a mixture of phenylene and oxyphenylene units, as shown in Scheme 1.

For the oxidation of glucose, oxygen is necessary as shown in Scheme 2. The reaction proceeded under neat oxygen gas as well as air, and the polymerization results were close to each other. These data indicate that the air atmosphere was sufficient to provide oxygen molecules with the reaction mixture. The polymerization in $80 \%$ dioxane resulted in the formation of the polymer in low yields (entries 1 and 2). By using 60\% dioxane, the polymer yield increased up to around $70 \%$, whereas the DSP value and the molecular weight of the DMF-soluble part decreased (entries 3-7). The $\mathrm{pH}$ of buffers scarcely affected the polymerization results.

Next, the effect of the type of an organic solvent has been examined in a mixture of succinate buffer ( $\mathrm{pH} 5.5)$ $(60: 40 \%)$ under air (Table II). The insoluble polymer was obtained in an aqueous acetonitrile or acetone (entries 1 and 2). From other cases, the resulting polymer

Table I. HRP-catalyzed oxidative polymerization of phenol in the presence of glucose and glucose oxidase ${ }^{\mathrm{a}}$

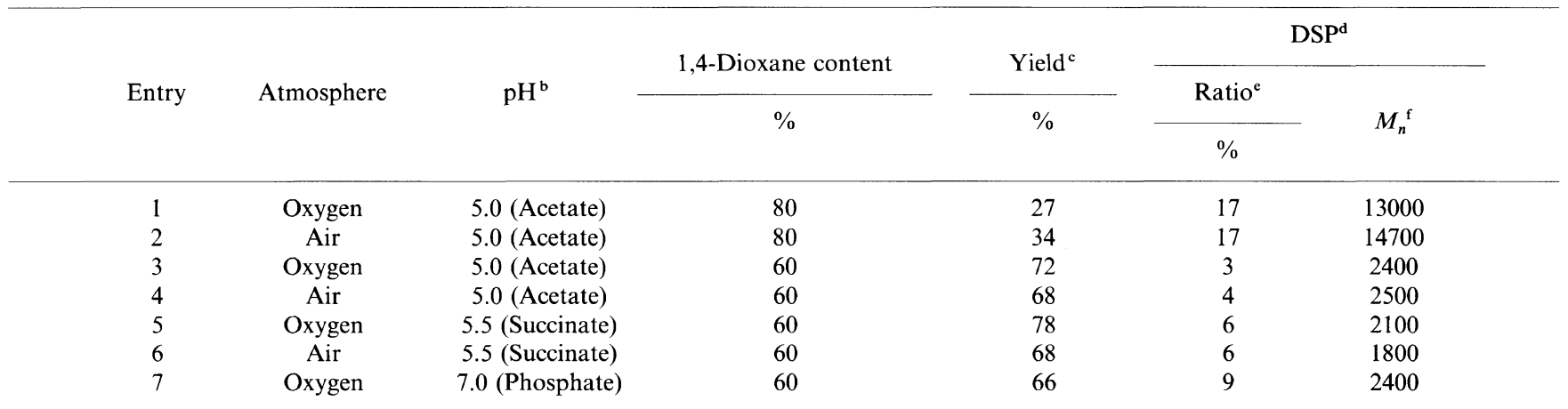

${ }^{a}$ Polymerization of phenol using HRP catalyst in the presence of glucose and glucose oxidase in a mixture of 1,4-dioxane and buffer at room temperature for $48 \mathrm{~h}$. ${ }^{\mathrm{b}}$ In parenthesis, buffer salt. ${ }^{\mathrm{c}}$ Methanol-insoluble part. ${ }^{\mathrm{d}}$ DMF-soluble part of polymer. ${ }^{\mathrm{e}}$ Ratio of DSP in isolated polymer. ${ }^{\mathrm{f}}$ Determined by GPC.

Table II. Effects of solvent composition on bienzymatic catalysis systems ${ }^{\text {a }}$

\begin{tabular}{|c|c|c|c|c|}
\hline \multirow{3}{*}{ Entry } & \multirow{3}{*}{ Solvent composition ${ }^{b}$} & \multirow{3}{*}{$\frac{\text { Yield }^{\mathrm{c}}}{\%}$} & \multicolumn{2}{|c|}{$\operatorname{DSP}^{d}$} \\
\hline & & & Katio & $M \mathrm{f}$ \\
\hline & & & $\%$ & \\
\hline 1 & Acetonitrile (15)/Buffer (10) & 45 & 0 & - \\
\hline 2 & Acetone (15)/Buffer (10) & 57 & 0 & - \\
\hline 4 & Ethanol (15)/Buffer (10) & 61 & 12 & 6600 \\
\hline 5 & Ethyl acetate (15)/Buffer (10) & 42 & 29 & 7100 \\
\hline 6 & Methanol (15)/Buffer (10) & 15 & 78 & 7200 \\
\hline 7 & iso-Propanol (15)/Buffer (10) & 51 & 2 & 7500 \\
\hline 8 & 1,4-Dioxane (10)/Ethyl acetate (5)/Buffer (10) & 62 & 13 & 4400 \\
\hline 9 & 1,4-Dioxane (7.5)/Ethyl acetate (7.5)/Buffer (10) & 64 & 69 & 7000 \\
\hline 10 & 1,4-Dioxane (5)/Ethyl acetate (10)/Buffer (10) & 56 & 72 & 7500 \\
\hline
\end{tabular}

${ }^{a}$ Polymerization of phenol using HRP catalyst in the presence of glucose and glucose oxidase in a mixture of an organic solvent and succinate buffer ( $\mathrm{pH} 5.5)$ at room temperature for $48 \mathrm{~h}$ under air. ${ }^{\mathrm{b}} \mathrm{In}$ parenthesis, solvent volumes in mL. ${ }^{\mathrm{c}} \mathrm{Methanol-insoluble} \mathrm{part.}$ ${ }^{\mathrm{d}}$ DMF-soluble part of polymer. ${ }^{\mathrm{e}}$ Ratio of DSP in isolated polymer. ${ }^{\mathrm{f}}$ Determined by GPC. 
Table III. HRP-catalyzed oxidative polymerization of $p$-alkylphenol in the presence of glucose and glucose oxidase $^{a}$

\begin{tabular}{|c|c|c|c|c|}
\hline \multirow{3}{*}{ Entry } & \multirow{3}{*}{ Monomer } & \multirow{3}{*}{$\frac{\text { Yield }^{\mathrm{b}}}{\%}$} & \multicolumn{2}{|c|}{$\operatorname{DSP}^{\mathrm{c}}$} \\
\hline & & & Ratio $^{d}$ & \\
\hline & & & $\%$ & \\
\hline 1 & $p$-Cresol & 26 & 50 & 4400 \\
\hline 2 & $p$-Ethylphenol & 57 & 38 & 4300 \\
\hline 3 & $p$-n-Propylphenol & 64 & 36 & 3800 \\
\hline 4 & $p$-n-Butylphenol & 65 & 36 & 4200 \\
\hline 5 & $p$ - $n$-Pentylphenol & 72 & 55 & 3200 \\
\hline
\end{tabular}

a Polymerization of $p$-alkylphenol using HRP catalyst in the presence of glucose and glucose oxidase in a mixture of 1,4-dioxane and succinate buffer $(\mathrm{pH} 5.5)(60: 40 \mathrm{vol} \%)$ at room temperature for $48 \mathrm{~h}$ under air. ${ }^{\mathrm{b}}$ Methanol-insoluble part. ${ }^{\mathrm{c}} \mathrm{DMF}$-soluble part of polymer. ${ }^{d}$ Ratio of DSP in isolated polymer. ${ }^{\mathrm{e}}$ Determined by GPC.

was partly soluble in DMF, the molecular weight of DSP was around $7 \times 10^{3}$ except that obtained in the aqueous 1,4-dioxane. The polymerization using ethanol or $i$ propanol produced the polymer showing low solubility toward DMF (entries 4 and 7). The aqueous methanol solvent improved the solubility up to $78 \%$, however, the polymer yield was very low (entry 6).

The polymerization in the aqueous 1,4-dioxane produced the polymer in a moderate yield, however, its solubility and molecular weight were low. Then, the mixed solvent of 1,4-dioxane, ethyl acetate, and the succinate buffer has been employed for the polymerization (entries 8-10). In using more than $7.5 \mathrm{~mL}$ of ethyl acetate (entries 9 and 10), the DSP value increased, comparable with those obtained in the binary solvent system (entries 3 and 5).

The present bienzymatic catalysis also induced the oxidative polymerization of $p$-alkylphenols. The polymer yield increased with increasing the alkyl chain length. ${ }^{6}$ A similar behavior was observed in the HRP-catalyzed polymerization using a hydrogen peroxide reagent as oxidizing agent. The DSP value and molecular weight of the resulting polymer did not depend on the chain length of the substituent.

Acknowledgments. This work was partly supported by a Grant-in-Aid for Specially Promoted Research (No. 08102002) from the Ministry of Education, Science, and Culture of Japan and by Proposal Based Advanced Industrial Technology R \& D Program. H. K. gratefully acknowledges the receipt of a scholarship from the Japan Society for Promotion of Science (JSPS).

\section{REFERENCES AND NOTES}

1. S. Kobayashi, S. Shoda, and H. Uyama, Adv. Polym. Sci., 21, 1 (1995).

2. H. Ritter, Trends Polym. Sci., 1, 171 (1993).

3. J. S. Dordick, M. A. Marletta, and A. M. Klibanov, Biotechnol. Bioeng., 30, 31 (1987).

4. J. A. Akkara, K. J. Senecal, and D. L. Kaplan, J. Polym. Sci., Polym. Chem. Ed., 29, 1561 (1991).

5. H. Uyama, H. Kurioka, I. Kaneko, and S. Kobayashi, Chem. Lett., 423 (1994).

6. H. Kurioka, I. Komatsu, H. Uyama, and S. Kobayashi, Macromol. Rapid Commun., 15, 507 (1994).

7. H. Uyama, H. Kurioka, I. Komatsu, J. Sugihara, and S. Kobayashi, Macromol. Reports, A32, 649 (1995).

8. H. Uyama, H. Kurioka, J. Sugihara, I. Komatsu, and S. Kobayashi, Bull. Chem. Soc. Jpn., 68, 3209 (1995).

9. H. Uyama, H. Kurioka, J. Sugihara, and S. Kobayashi, Bull. Chem. Soc. Jpn., 69, 189 (1996).

10. M. S. Ayyagari, K. A. Marx, S. K. Tripathy, J. A. Akkara, and D. L. Kaplan, Macromolecules, 28, 5192 (1995).

11. L. Wang, E. Kobatake, Y. Ikariyama, and M. Aizawa, J. Polym. Sci., Polym. Chem. Ed., 31, 2855 (1993).

12. R. Ikeda, H. Uyama, and S. Kobayashi, Macromolecules, 29, 3053 (1996).

13. P. W. Kopf, "Encyclopedia of Polymer Science and Engineering," Vol. 11, 2nd ed, John Wiley \& Sons, New York, N.Y., 1986, pp 45-95.

14. R. Z. Kazandjian, J. S. Dordick, and A. M. Klibanov, Biotechnol. Bioeng., 28, 417 (1986).

15. G. G. Guilbault, "Handbook of Enzymatic Methods of Analysis," Marcel Dekker, New York, N.Y., 1976. 